\title{
Traduire
}

Revue française de la traduction

\section{Le Mot d'Or de la Traduction francophone}

Jean Marcel Lauginie

\section{(2) OpenEdition \\ Journals}

Édition électronique

URL : http://journals.openedition.org/traduire/220

DOI : $10.4000 /$ traduire.220

ISSN : 2272-9992

Éditeur

Société française des traducteurs

Édition imprimée

Date de publication : 1 juin 2011

Pagination : 129-134

ISSN : 0395-773X

\section{Référence électronique}

Jean Marcel Lauginie, «Le Mot d'Or de la Traduction francophone », Traduire [En ligne], 224 | 2011, mis en ligne le 03 février 2014, consulté le 30 avril 2019. URL : http://journals.openedition.org/traduire/220 ; DOI : 10.4000/traduire.220 


\section{Le Mot d'Or de la Traduction francophone}

Jean Marcel Lauginie

Merci à la revue Traduire de nous permettre de raconter à ses lecteurs l'histoire d'une action heureuse qui n'aurait pu naître et se développer sans la SFT.

\section{Une naissance tardive, mais sous d'heureux auspices !}

Né en 2003, Le Mot d'Or de la Traduction francophone avait été précédé, dès 1989, par les Mots d'Or des apprenants et des professionnels décernés par notre association Actions pour promouvoir le français des affaires (APFA), placée sous le patronage de la Délégation générale à la langue française et aux langues de France (DGLFLF), de l'Organisation internationale de la Francophonie (OIF) et de l'Union internationale de la Presse francophone (UPF).

Les Mots d'Or saluent, au cœur de la mercatique terminologique, la volonté d'entreprendre fondée sur la créativité dans la maîtrise du vocabulaire des affaires en français et dans chaque langue maternelle.

Parmi les mécènes des Mots d'Or, le Centre d'échange d'informations pour la traduction littéraire de l'UNESCO remettait chaque année aux lauréats, grâce à notre interlocuteur Mauro Rosi, des ouvrages de la "Collection UNESCO d'œuvres représentatives".

Le 23 février 2003, lors d'une remise de Mots d'Or au siège de l'Agence intergouvernementale de la Francophonie (AIF), Mauro Rosi m'interpella ainsi " Que penseriez-vous d'un Mot d'Or de la Traduction? ", idée lumineuse soumise immédiatement au Directeur des Langues et de l'Écrit à l'AIF, Isidore Ndawel E Nziem, et formulée ainsi par Mauro Rosi : "La possibilité d'encourager la traduction littéraire en s'insérant dans le dispositif des Mots d'Or par la création d'un nouveau volet, placé sous le patronage de l'AlF ".

Cette initiative ne pouvait que nous ravir, car nous avions le sentiment, depuis quelques années, que les traducteurs auraient dû être depuis longtemps des interlocuteurs privilégiés de nos actions terminologiques dans le monde des affaires. 


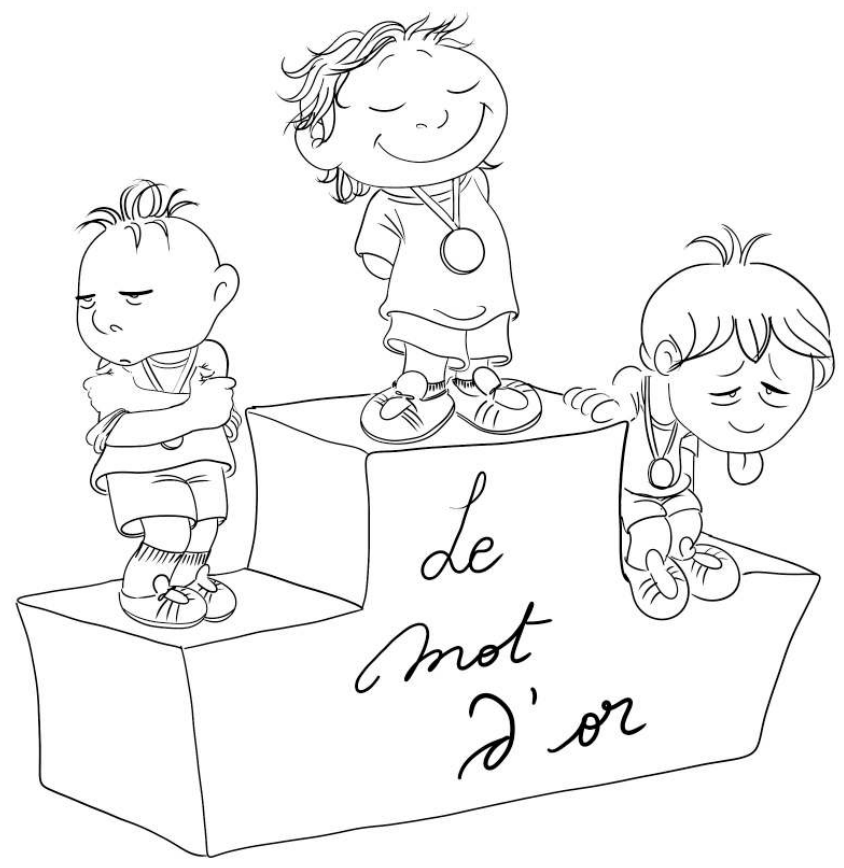

Illustration : Marlène Junius, http://alotoftralala.over-blog.com

Une première réunion eut lieu le 1er juillet 2003 pour bâtir un projet de telle sorte que le premier Mot d'Or de la Traduction francophone puisse être remis lors de la 16e Cérémonie des Mots d'Or au cours de nos Journées du français des affaires des 20/21 novembre 2003.

Or, heureux concours de circonstances, début juillet 2003, j'étais informé par une adhérente de l'APFA, Marie-Christine Lemasson, Déléguée de la Société Française des Traducteurs (SFT) auprès de la Fédération Internationale des Traducteurs (FIT), de la tenue à l'UNESCO du 50e anniversaire de la FIT également en novembre 2003 du 20 au 22 !

D'où ma proposition à Mauro Rosi, à Isidore Ndawel E Nziem, à Marie-Christine Lemasson et à Maria Angeles Lebret-Sanchez, Présidente de la SFT, de lancer le premier Mot d'Or de la Traduction francophone à l'UNESCO au cours du 50e anniversaire de la FIT.

La première réunion préparatoire se tint à la DGLFLF représentée par Pierre Janin, Inspecteur général de l'action culturelle, le 22 octobre 2003 et la réunion de délibération du jury, le 
5 novembre. Les pionnières représentant la SFT à ce premier jury furent : Suzanne Boizard et Marie-Christine Garcin.

L'heureuse élue de ce premier Mot d'Or de la Traduction francophone, Françoise Naour, pour sa traduction chinois-français du roman Des yeux gris clairs par Wang Meng aux Éditions Bleu de Chine, dont le talent nous a fait pénétrer dans la civilisation des Ouïghours ; Françoise Naour reçut le 21 novembre 2003 à l'UNESCO la Médaille des Mots d'Or, œuvre d'Henri Chopin et de la Monnaie de Paris.

\section{Le jury, sa composition, les critères retenus}

En 2005, avec l'entrée de l'Union internationale de la Presse francophone (UPF), représentée par Alain Garnier, le Jury du Mot d'Or de la Traduction francophone prend sa forme actuelle.

Le jury est ainsi composé en 2011 de membres de la SFT, Suzanne Boizard et Françoise De Dax, de représentants de l'Organisation internationale de la Francophonie (OIF) (I'ancienne AIF) et de la DGLFLF, d'Alain Garnier membre de I'UPF et de membres de notre association, l'APFA. Le Centre d'échange d'informations pour la traduction littéraire de l'UNESCO, ayant laissé la place à de nouvelles structures, s'est retiré.

Le jury composé de 5 à 7 membres, qui participent régulièrement aux réunions de lecture et de choix, est d'une taille idéale pour un groupe de travail, ce qui contribue indéniablement au bon climat de nos délibérations.

Comme pour tout jury, les appréciations dépendent des critères de choix retenus. Aux critères de base pour apprécier une traduction vers le français :

- fidélité et qualité de la traduction,

- qualité littéraire du texte français,

le jury a ajouté deux critères spécifiques du monde des Mots d'Or, c'est-à-dire du monde des affaires et de l'économie au sens large :

- valeur de découverte de sociétés et de cultures inconnues ou méconnues,

- aspects socio-économiques et terminologiques.

Ce quatrième critère avait été placé, en 2003, au sein du premier critère.

Durant ces huit années d'existence, ces quatre critères se sont avérés très efficaces et d'un emploi aisé : ils nous ont permis de sélectionner facilement, au cours de trois puis cinq réunions annuelles, la meilleure traduction parmi une dizaine d'ouvrages proposés par les membres du jury et, à travers eux, des membres des organisations représentées. 


\section{Le développement du Mot d'Or de la Traduction francophone}

Il est bien illustré par le palmarès ; après Françoise Naour en 2003, le Mot d'Or de la Traduction francophone revenait :

en 2004, à Veronika Nentcheva et Éric Naulleau pour nous avoir offert une remarquable découverte en français du lumineux roman bulgare, Abraham le Poivrot, d'Angel Wagenstein aux éditions L'Esprit des péninsules ;

en 2005, à Doan Cam Thi pour son esprit d'entreprise qui l'a conduit à prendre l'initiative de réunir, traduire et présenter des récits vietnamiens, Au rez-de-chaussée du paradis, aux éditions Philippe Picquier, ainsi que pour sa capacité à épouser brillamment quatorze styles différents représentatifs de la jeune littérature vietnamienne, engagée pour partie, dans le monde économique ;

en 2007 (pour 2006), à Rania Samara pour sa traduction de l'arabe (Égypte) du roman de Naguib Mahfouz, Son Excellence, publié en langue arabe en 1976, édité en 2006 chez SinbadActes Sud ; Rania Samara a su souligner le regard lucide plein d'humour, porté par Naguib Mahfouz, sur l'irrésistible ascension d'un employé dans un système bureaucratique ;

en 2008, à Mooshegh Abrahamian pour la première traduction de l'arménien du roman de Raffi écrit en 1880, Le Fou, édité en 2007 chez Bleu autour ; Mooshegh Abrahamian a su évoquer dans ce roman très riche aux multiples personnages, en un style vif attentif aux néologismes qui emporte le lecteur, la vie quotidienne d'une société broyée par les drames de l'histoire mais dont la vision d'avenir reste portée par un humanisme fondé sur le progrès technique, l'instruction et l'enrichissement de la langue maternelle ;

en 2009, il a été attribué à Yan Hansheng et Lisa Carducci pour avoir permis une excellente découverte en français du roman du chinois Jiang Rong Le Totem du loup, édité chez François Bourin. Grâce à la force de leur traduction, Yan Hansheng et Lisa Carducci offrent à la francophonie une démonstration sensible, brillante et limpide du principe du "juste milieu ", à l'opposé de la " quantité d'abord! ", si nécessaire en ce temps de crise mondiale. Leur traduction parfaite permet déjà au vieux pasteur nomade et au jeune instruit d'éclairer les décisions pour la survie des civilisations ;

en 2010, à Philippe Vigreux pour sa traduction de l'arabe (Libye) du roman d'Ibrahim Al-Koni, Comme un appel du lointain, édité chez Michel de Maule. Grâce au lyrisme et à la beauté de sa traduction, Philippe Vigreux offre à la francophonie toute la force et la vérité profonde de la civilisation du désert où les techniques marchandes, nommées avec les termes justes du " troc à distance " aux " enchères inversées ", sont présentes ;

et, en 2011, à Anne Colin du Terrail pour avoir permis de pénétrer en français dans l'âme et I'histoire finlandaises, au siècle dernier, saisies par Arto Paasilinna dans son roman Sang chaud, nerfs d'acier, aux Éditions Denoël. Le jury a été enchanté par la beauté littéraire de la traduction 
dès la première phrase du roman, par la précision des descriptions, par l'emploi du terme juste tel que l'avitaillement ou le bail emphytéotique, par l'art remarquable d'Anne Colin du Terrail pour rendre compte de situations cocasses à l'image de cette éducation commerciale, pétrie d'humanité, où l'honnêteté côtoie la malice et par son attention permanente au sens des mots, à leur étymologie et aux langues.

Ainsi, depuis 2003, le palmarès a honoré les traducteurs de deux romans chinois, d'un roman bulgare, d'un recueil de récits vietnamiens, d'un roman égyptien, d'un roman arménien, d'un roman libyien et d'un roman finlandais.

Cette diversité des langues et des pays est en accord avec la raison d'être du Mot d'Or de la Traduction francophone que nous formulons ainsi : un signe de reconnaissance du travail des traducteurs vers la langue française en faveur de la découverte d'autres cultures, notamment sous leurs aspects linguistiques et socio-économiques.

\section{Quel avenir ?}

Le Mot d'Or de la Traduction francophone est d'abord un encouragement à traduire vers le français.

Mais, comme nous venons de le souligner, il veut aussi agir pour le maintien et le renforcement de la diversité des langues de la francophonie dans la vie économique quotidienne.

À cet égard, nous manquons de propositions d'ouvrages écrits dans certaines de ces langues, par exemple, le bambara, le wolof ou un des nombreux créoles, et traduits en français. Nous sommes prêts à retenir des manuscrits non encore publiés, qu'il s'agisse de romans ou d'essais.

Nous serions très heureux de recevoir des conseils et des informations des traducteurs de la SFT pour mieux réaliser cet objectif.

Jean Marcel Lauginie, président du Jury des Mots d'Or

Courriel : apfa.lemotdor@orange.fr

Site : www.presse-francophone.org/apfa ou www.apfa.asso.fr 
Jean Marcel LAUGINIE, président créateur, en 1984, de l'association Actions pour promouvoir le français des affaires (APFA), sous le patronage de la Délégation générale à la langue française et aux langues de France, de l'Organisation internationale de la Francophonie et de l'Union internationale de la Presse francophone.

Agrégé des techniques économiques de gestion, membre de le Commission spécialisée de terminologie et de néologie en matière économique et financière.

\section{Bibliographie}

APFA, 2000 mots d'or du français des affaires, Orléans, CRDP-SCÉRÉN du Centre, Collection Loïc Depecker.

APFA, mots d'or transfrontières de la francophonie, Langues des affaires transnationales d'Afrique, transfrontalières de France, Orléans, CRDP-SCÉRÉN du Centre, Collection Loïc Depecker.

APFA, Mots-clés des affaires pour l'Europe des 27, Orléans, CRDP-SCÉRÉN du Centre, Collection Loïc Depecker.

APFA, CONSULTEZ LES MOTS DES AFFAIRES EN 53 LANGUES, www.presse-francophone.org/apfa, consulté le 20 avril 2011. 